\title{
Body image and compulsive exercise: are there associations with depression among university students?
}

\author{
Klara Edlund $^{1,2}$ (D) Fred Johansson ${ }^{1,2} \cdot$ Rebecca Lindroth $^{3} \cdot$ Louise Bergman $^{3} \cdot$ Tobias Sundberg $^{1,2} \cdot$ Eva Skillgate $^{1,2}$
}

Received: 5 October 2021 / Accepted: 29 January 2022 / Published online: 18 February 2022

(c) The Author(s) 2022

\begin{abstract}
Purpose Mental health problems among university students have been reported to be significantly increasing and suggested to be associated with college drop-out. Body dissatisfaction and compulsive exercise are both constructs relevant for mental health problems in general and eating disorders in particular. This study examined associations between body dissatisfaction, compulsive exercise and self-reported symptoms of depression among Swedish university students.

Methods Participants $(n=4262)$ are students in an ongoing cohort study, and data from the baseline assessment were used. Four linear regression models were built to explore the associations between body dissatisfaction, compulsive weight control exercise and depressive symptoms.

Results Our findings showed that females reported higher levels of body dissatisfaction than males. Body dissatisfaction and compulsive exercise were associated with self-reported symptoms of depression in this non-clinical population. Results showed that compulsive exercise was negatively associated with reported symptoms of depression, while body dissatisfaction was positively associated with symptoms of depression.

Conclusion In line with previous research, there was a gender difference in body dissatisfaction where females displayed higher levels of dissatisfaction than males. Body dissatisfaction was positively associated with reported symptoms of depression, suggesting support of previous research indicating body dissatisfaction to increase mental health problems. Compulsive exercise was negatively associated with symptoms of depression suggesting a behavior negatively reinforced, supporting both constructs to be of interest for reported symptoms of depression in a non-clinical population of Swedish university students. Level of evidence III, cohort study.
\end{abstract}

Trial registration http://clinicaltrials.gov/ID: NCT04465435.

Keywords Body dissatisfaction $\cdot$ Compulsive exercise $\cdot$ Depression $\cdot$ University students

Klara Edlund

Klara.edlund@ki.se; klara.edlund@shh.se

1 Musculoskeletal and Sports Injury Epidemiology Center, Department of Health Promotion Sciences, Sophiahemmet University, Sophiahemmet Högskola, Box 5605, 11486 Stockholm, Sweden

2 Unit of Intervention and Implementation Research on Worker Health, Institute of Environmental Medicine, Karolinska Institutet, Stockholm, Sweden

3 Department of Psychology, Uppsala University, Uppsala, Sweden

\section{Introduction}

Mental health problems among university students have been reported to be significantly increasing, impacting health and wellbeing $[25,30]$, and suggested to be associated with college drop-out [3]. High levels of psychological distress including anxiety, stress and depression have been well documented among medical students [13] and body dissatisfaction [41] and compulsive exercise [60] may be factors associated with this psychological stress.

Body image has been described as a multidimensional construct that encompasses the internalized view one has of one's body, including perceptions, thoughts, feelings, and attitudes related to physical aspects of the body, such as weight and shape [58]. Body dissatisfaction (negative attitudes toward the own physical appearance) is one aspect of 
body image [23]. Most research on body image and body dissatisfaction in the past 20 years has primarily focused on females' idealization of a thin body [45]. However, during the past decade, male body image issues have drawn more attention $[5,39]$. The ideal male body is usually characterized be lean muscularity, leading to concerns generally related to being too fat or not muscular enough [44]. A recent meta-analysis by Barnes et al. [4] demonstrated that an association between male body dissatisfaction and anxiety and depression is likely to exist. Muscle tone along with thinness is also a focus for females resulting in concerns about weight, shape, and muscularity [21, 28, 62]. Body dissatisfaction has been strongly linked to eating disorder symptomatology as well as eating disorder relapse [24]. Further, body dissatisfaction has been shown to be related to extreme behaviors with adverse effects, such as restrained eating, purging, and over-exercising [45], and is considered a risk factor for eating disorders [34, 40, 47, 48], a series of risky health behaviors, such as alcohol and drug use, gambling, self-harm [9], and poor psychological health [11, 17, 40].

Empirical longitudinal studies have confirmed that body dissatisfaction predicts depression among females $[6,16,17$, $22,38,42,50]$. Most of these studies were carried out in the early 2000s with participants born in the 1980s. Since body dissatisfaction is increasing over time [54, 57], it is crucial to explore the Millennial generations due to the important role of internet, technology, and social media on lifestyles of more recent generations [8]. The study by Bornioli and colleagues [8] lends support to previous theoretical ideas proposed by Stice and Bearman $[6,49,50]$ that young women might suffer adverse psychological health conditions due to their dissatisfaction with their body. Further, it was demonstrated in the Bornioli study [8] that the relationship between body dissatisfaction and depression persisted with control for BMI, indicating that body image is a multi-dimensional construct, not determined exclusively by BMI.

As mentioned above, body dissatisfaction has been identified as a risk factor for disordered eating. Furthermore, has body dissatisfaction also been demonstrated to predict later onset of depressive episodes in adolescence [8]. Body dissatisfaction has been shown to be associated with both depression and anxiety in Swedish adolescents [26], and young female adults [52]. Further, symptoms of depression and other mental health symptoms have been shown to be associated with suicidal ideation among UK university students [1]. Sub-threshold symptoms of eating disorders among college students have also been shown to be highly predictive of suicidality [32]. Further, The Healthy Minds Study [32], the largest mental health survey of college populations in the United States with data from 71712 students randomly selected from 77 campuses, have reported that weight and shape concerns were associated with higher rates of suicidality, even after controlling for psychiatric comorbidity. Thus, body dissatisfaction is closely linked to disturbed eating and depression, among university students and it has been argued that the pervasive issues of body dissatisfaction are public health concerns [8].

Compulsive exercise is characterized by craving for exercise and training along with weight and shape concerns, resulting in uncontrollable excessive behaviors with harmful consequences. There is a persistent continuation to both alleviate withdrawal symptoms (extreme guilt and/ or negative affect when unable to exercise) and to avoid the perceived negative consequences of not exercising [35]. Injuries and impaired social relations are some of the harmful health consequences along with eating disorders pathology and depression [31]. Thus, excessive, and compulsive exercise represents a rigidity and an extreme urge to exercise, even when one does not find joy in it [55], and it has been clearly linked to eating pathology [18]. Research assessing the prevalence of compulsive exercise in non-clinical samples, particularly among college students is lacking, although a recent study has reported compulsive exercise to be relatively common among college students and to be associated with poor mental health symptoms and substance use [20]. Among college students, engaging in compulsive exercise has been reported to be associated with greater risk of a concurrent eating disorder [46], and compulsive exercise is broadly conceptualized as a central component in the development of eating disorder symptoms $[36,37]$. As previously presented in the literature, body dissatisfaction and compulsive exercise have been linked to mental health problems (e.g., [52], Stroeber and Otto 2006; [60].

The present study is part of an ongoing large prospective cohort study of mental health problems and musculoskeletal pain in university students in Sweden (see below; SUN; http://clinicaltrials.gov/ID: NCT04465435). Several variables are included in the study, such as life-style factors, different aspects of health, and potential problem behaviors, such as substance use and gambling for money, which are planned for upcoming presentations. Body dissatisfaction and compulsive exercise are two factors studied, with the hypothesis, based on the literature, that they will be linked to self-reported symptoms of depression among students. Assessing body dissatisfaction and compulsive exercise to establish whether there is an association with depression in this non-clinical population is a somewhat novel approach that could be of importance for future causal analysis to better understand how these constructs play a part in depression among students. Thus, the aim of this study was to explore the associations between compulsive exercise, body dissatisfaction and self-reported symptoms of depression in a large sample of Swedish university students. 


\section{Materials and methods}

\section{Source population, inclusion criteria, recruitment and data collection}

The Sustainable UNiversity life study, the SUN-study, is a cohort study of undergraduate- and graduate (up to master level) university students in Sweden. Eligible for participation include students enrolled in full-time educational programs with at least one academic year before planned graduation. This study include 4262 students using baseline data from the SUN-study (http://clinicaltrials.gov/ ID: NCT04465435; Edlund et al., submitted) [14]. The overall aim of the SUN-study is to advance the knowledge about mental health problems and musculoskeletal pain in university students. The source population are students at universities/colleges in the greater Stockholm area and Örebro, attending selected educational programs in health, nursing, medical, and social sciences as well as technology. The universities selected were located close to the work location of the research since the research staffs' physical presence was an important recruitment strategy for the study.

Students eligible for the study were mainly invited through e-mail. Email addresses were distributed through the universities. All participants provided online informed consent to participate in the study, after receiving online and/or in person information about the purpose and procedure of the study, and that it has been approved by the Swedish Ethical Review Authority (Reference Number: 2019-03276; 2020-01449). Data were collected online with a baseline questionnaire, followed by four follow-up questionnaires sent out every three months during one academic year. The first baseline questionnaire took about $30 \mathrm{~min}$ to complete and the follow-up questionnaires somewhere between 10 and $15 \mathrm{~min}$. As a gesture of appreciation, all students participating in the study were offered a one-month free pass to one of the major health club chains in Sweden, ACTIC, to whom a collaboration for sponsorship has been established. The online platforms used for the data collection were SUNET, hosted online by SUNET Artologik, a secure web-based survey system used in Swedish higher education [53]. The system has previously been used by members of our research group [19].

\section{Instruments}

Depression anxiety scale (DASS-21) The depression anxiety stress scale is a self-report questionnaire used to assess self-reported symptoms of mental health problems. For the present study, only the scale measuring depression was used (DASS-21; [33]. The original DASS consists of 42 items, addressing three different dimensions of symptoms (depression, anxiety and stress) within the last week. The version most widely used is the adapted and shortened version with 21 items, which is used for the present study. DASS-21 has reported adequate psychometric properties (test-retest and internal consistency, a 0.81-0.96 [2,33], and has been developed to measure symptoms of depression, anxiety and stress in both clinical and non-clinical populations. The Cronbach's for the depression-subscale $\alpha$ was 0.91 in this sample.

Compulsive exercise test (CET) The compulsive exercise test is a self-report questionnaire designed to explore the emotional, cognitive, and behavioral characteristics of compulsive exercise (CET; [55]. It comprises 24 items answered on a Likert scale from 0 (never true) to 5 (always true). The CET consists of five subscales: "avoidance and rule-driven behavior", "weight control exercise", "mood improvement", "lack of exercise enjoyment" and "exercise rigidity". Two subscales are included in the study: "Weight control exercise" (WCE) and "Avoidance of negative affect and rule-driven behavior" (ARDB), since these subscales have adequate validity (factor analysis) and internal consistency (WCE: $\mathrm{a}=0.82$, avoidance and rule-driven behavior ARDB: $\mathrm{a}=0.87$,Plateau et al., 2014). A mean score of $\geq 3.47$ for the WCE scale and $\geq 2.75$ for ARDB have been reported in a Norwegian sample of eating disorders patients [59]. For the present study, Cronbach's alpha was 0.83 for WCE and 0.90 for ARDB. The questions assess behaviors for weight loss and emotion regulation using training.

Body shape questionnaire (BSQ-8C). The brief version of the Body Shape Questionnaire (BSQ-8C, 8-item version; [61], based on the full 34-item version [10], was used to assess body dissatisfaction. The questions are reflecting the past four weeks regarding body shape concerns with questions addressing e.g., fear of becoming fat, self-consciousness about shape when in company with others and feelings of being excessively large and rounded. A score $<19$ indicates no concern with shape, between 19 and 25 mild concern, 26-33 moderate concern and $>33$ marked concerns with shape. The eight-item version of BSQ has been found to be sufficiently reliable and valid [15], and the short versions capture the one-factor structure of the full version BSQ [29]. Welch and colleagues (2012) used the BSQ-8C Swedish version as a stand-alone measurement of body dissatisfaction and found it to show high internal consistency of $a=0.94$, excellent test-retest properties. In the present study population, Cronbach's alpha was 0.93 .

\section{Statistical analysis}

Spearman's correlations between BSQ and the subscales of CET were calculated. Cohen's $d$ was used as a measure of effect size. Four linear regression models were built 
to explore the associations between body dissatisfaction, compulsive weight control exercise and depressive symptoms. The first two models explored bivariate associations by regressing depressive symptoms on body dissatisfaction and compulsive weight control exercise, respectively. The third model included both body dissatisfaction and compulsive weight control exercise to explore each of the independent variables' association to depression while holding the other constant. In the fourth model, an interaction-term between compulsive weight control exercise and body dissatisfaction was added to explore whether body dissatisfaction modified the association between compulsive weight control exercise and depressive symptoms. All regression models were adjusted for gender (male vs not male), age (continuous) and level of parental education (at least one parent with university education vs no parent with university education). The BSQ, CET and DASS-21 were all treated as continuous variables in the regression models. Multicollinearity was assessed by the variance inflation factor (VIF). A VIF below ten was regarded as satisfactory.

\section{Ethical considerations}

Prior to the data collection, the Universities' Educational Support Offices and Student Health Services were informed about the study and granted approval to implement the study at each site. Before inclusion in the study, informed consent was given by all participants. The study was approved by the Swedish Ethical Review Authority (Reference Number: 2019-03276; 2020-01449).

\section{Results}

A total of 18,973 students were invited to the study and 4263 (23\%) participated by completing the web-based survey. See Table 1 for sample demographics.

\section{Body dissatisfaction}

Recalculated scoring for BSQ-8C, based on Taylor [56] is shown in Table 2.

On average, female participants scored higher on the BSQ (mean $(\mathrm{M})=21.3, \mathrm{SD}=10.2, \mathrm{SE}=0.2$ ), than male participants $(\mathrm{M}=14.7, \mathrm{SD}=7.1, \mathrm{SE}=0.2)$. This difference, 6. 7, 95\% CI [6.2, 7.2], was significant $t(4153.5)=25.1$, $p<0.0001$; with a medium to large effect size, $d=0.7$. Thus, women reported higher levels of body dissatisfaction than men.

\section{Compulsive exercise}

On average, females scored higher on Weight Control Exercise $(\mathrm{M}=6.6, \mathrm{SD}=4.9, \mathrm{SE}=0.1)$, than males $(\mathrm{M}=5.2, \mathrm{SD}=4.1, \mathrm{SE}=0.1)$. This difference, $1.4,95 \%$
Table 1 Characteristics of full sample and divided by males and females

\begin{tabular}{llll}
\hline & $\begin{array}{l}\text { Full sample } \\
(n=4263)\end{array}$ & $\begin{array}{l}\text { Males } \\
(n=1592)\end{array}$ & Females $(n=2645)$ \\
\hline $\begin{array}{l}\text { Age, M (SD) } \\
\text { Gender, } n(\%)\end{array}$ & $24.6(6.1)$ & $23.8(5.4)$ & $25.1(6.4)$ \\
Female & $2645(62 \%)$ & - & - \\
Male & $1592(37 \%)$ & - & - \\
Other & $26(1 \%)$ & & \\
Year of study & & & \\
1st & $1733(41 \%)$ & $614(39 \%)$ & $1108(42 \%)$ \\
2nd & $936(22 \%)$ & $320(20 \%)$ & $608(23 \%)$ \\
3rd & $659(15 \%)$ & $256(16 \%)$ & $399(15 \%)$ \\
Masters & $935(22 \%)$ & $402(25 \%)$ & $530(20 \%)$ \\
Type of education, & & & \\
$n(\%)$ & & & \\
Medical & $1966(46 \%)$ & $458(29 \%)$ & $1493(56 \%)$ \\
Technical & $1770(42 \%)$ & $956(60 \%)$ & $805(30 \%)$ \\
Social sciences & $527(12 \%)$ & $178(11 \%)$ & $347(13 \%)$ \\
Place of birth, $n(\%)$ & & & \\
Sweden & $3337(78 \%)$ & $1250(79 \%)$ & $2068(78 \%)$ \\
Nordic countries & $138(3 \%)$ & $35(2 \%)$ & $102(4 \%)$ \\
Europe & $263(6 \%)$ & $97(6 \%)$ & $163(6 \%)$ \\
Outside europe & $525(12 \%)$ & $210(13 \%)$ & $312(12 \%)$ \\
\hline
\end{tabular}

$S D$ standard deviation, $n$ number

Table 2 Percentage dispersion of levels of concern with shape $(n=4263)$

\begin{tabular}{lllll}
\hline & Score & $\begin{array}{l}\text { Total } \\
n=4263 \\
n,(\%)\end{array}$ & $\begin{array}{l}\text { Female } \\
n=2645 \\
n,(\%)\end{array}$ & $\begin{array}{l}\text { Male } \\
n=1592 \\
n,(\%)\end{array}$ \\
\hline No & $<19$ & $2635(62 \%)$ & $1337(51 \%)$ & $1285(81 \%)$ \\
Mild & $19-25$ & $664(16 \%)$ & $488(18 \%)$ & $173(11 \%)$ \\
Moderate & $26-33$ & $552(12 \%)$ & $426(16 \%)$ & $92(6 \%)$ \\
Marked & $>33$ & $442(10 \%)$ & $394(15 \%)$ & $42(3 \%)$ \\
\hline
\end{tabular}

CI $[1.2,1.7]$, was significant $t(3820.5)=10.2, p<0.0001$; although the effect size was small, $d=0.3$. Thus, a significant gender difference was found, where women more often self-reported to exercise for weight and shape reasons than men. On average, females also scored higher on Avoidance of Negative Affect and Rule-Driven Behavior $(\mathrm{M}=9.01, \mathrm{SD}=6.89, \mathrm{SE}=0.13)$ than males $(\mathrm{M}=8.36$, $\mathrm{SD}=6.63, \mathrm{SE}=0.17)$. This difference, $0.6595 \% \mathrm{CI}[0.24$; 1.07], was significant $t(3453.1)=3.06, p=0.002$, with a small effect size $d=0.1$. Moderate correlations were shown between body dissatisfaction and avoidance of negative affect and rule-driven behavior $(r=0.32)$ and finally, moderate correlations were found between avoidance of negative affect and rule-driven behavior and weight control exercise $(r=0.49)$, and between body dissatisfaction and weight control exercise $(r=0.64)$ (Table 3$)$. 
Table 3 Correlations between compulsive exercise and body dissatisfaction. Spearman's rank correlations with 95\% CI, $(n=4263)$

\begin{tabular}{llll}
\hline & BD* & WCE** & ARDB*** \\
\hline BD & 1 & & \\
WCE & $0.64(0.62-0.66)$ & 1 & \\
ARDB & $0.32(0.29-0.35)$ & $0.49(0.29-0.35)$ & 1 \\
\hline
\end{tabular}

* $B D$ body dissatisfaction

**WCE weight control exercise

***ARDB avoidance and rule-driven behavior

Table 4 Linear regression models of the association between compulsive training, body dissatisfaction, and depressive symptoms

\begin{tabular}{lllrl}
\hline & Beta & Std. error $t$ value $p$ value $95 \%$ CI \\
\hline $\begin{array}{lllll}\text { Model 1 (BD*) } \\
\text { BD }\end{array}$ & 0.18 & 0.21 & $6.84<0.001$ \\
$\begin{array}{l}\text { Model 2 (WCE**) } \\
\text { WCE }\end{array}$ & 0.16 & 0.02 & $10.34<0.001$ \\
$\begin{array}{l}\text { Model 3 (BD and } \\
\text { WCE) }\end{array}$ & & & & \\
BD & 0.23 & 0.01 & 23.77 & $<0.001$ \\
WCE & -0.15 & 0.02 & $-7.82<0.001$ \\
Model 4 (BD*WCE) & & & & \\
BD & 0.20 & 0.01 & $13.58<0.001$ \\
WCE & -0.26 & 0.04 & $-7.27<0.001$ \\
BD*WCE & 0.005 & 0.001 & $3.59<0.001$ \\
\hline
\end{tabular}

* $B D$ body dissatisfaction (Body Shape Questionnaire)

**WCE weight control exercise

\section{Depression}

On average, depression levels were higher among females $(\mathrm{M}=4.93 \mathrm{SD}=4.73, \mathrm{SE}=0.09)$ than males $(\mathrm{M}=4.42$ $\mathrm{SD}=4.65, \mathrm{SE}=0.12)$. This difference, $0.595 \% \mathrm{CI}[0.21$; 0.79 ] was significant $t(3396.7)=3.37, p=0.0007$ with a small effect size $d=0.1$.

\section{Association with reported symptoms of depression}

Table 4 shows the association between dissatisfaction (BD), weight control exercise (WCE) and level of depression, respectively. Model 1 showed that body dissatisfaction (BD) was positively associated with higher mean level of depression. Model 2 showed that also compulsive weight control exercise (WCE) was positively associated with mean level of depression. In model 3 (including both BD and WCE) the association between compulsive weight control exercise and depressive symptoms became negative, while the effect of body dissatisfaction on level of depression became more pronounced compared to model 1. Model 4 showed that there was a statistically significant interaction between body dissatisfaction and compulsive weight control exercise, although this interaction is too small to be considered practically significant.

Both BD and WCE were associated with depressive symptoms. When adding both predictors in the same model, WCE was negatively associated to depression symptoms, while BD was still positively associated. This indicates that within levels of BD, WCE is negatively associated to depression (Fig. 1).

\section{Discussion}

The aim of this study was to explore the associations between compulsive exercise, body dissatisfaction and selfreported symptoms of depression among Swedish university students.

\section{Results}

Our results confirmed previous research showing female students to display more body dissatisfaction than male students, with $31 \%$ of the females and $9 \%$ of the males reported moderate or marked levels of body dissatisfaction. Both body dissatisfaction and compulsive exercise were positively associated with self-reported symptoms of depression, when modeled separately. However, when modeled jointly, compulsive exercise showed a negative association with selfreported symptoms of depression, whereas body dissatisfaction showed a positive association. Regarding exercise for weight control and self-reported symptoms of depression, higher levels of weight control were related to lower symptoms of depression when adjusting for body dissatisfaction. Correlational analysis showed that all variables used in the multiple linear regression model were independent constructs. Since the correlations were found to be significant, even though the correlation not perfect, we argue that the constructs were reasonable to include in the model. Our results suggest body dissatisfaction and compulsive exercise to be associated with self-reported symptoms of depression among a non-clinical sample of university students.

Our results are in line with previous reports of excessive exercise being associated with different psychological traits where poor emotion regulation and compulsivity are two of them [12]. Previous studies have suggested compulsive exercise to be a dysfunctional emotion regulation strategy [43]. We suggest that these behaviors in the short run may serve as dysfunctional strategies, maintained by processes of negative reinforcement, where individuals continue to exercise as a result of the removal of aversive stimuli, such as negative emotions [7]. These results indicate that these variables are 
Fig. 1 Association between levels of BD, WCE and level of depression. The figure shows predicted values of depressive symptoms from model 4.The lines represent different levels of $\mathrm{BD}$, see legend

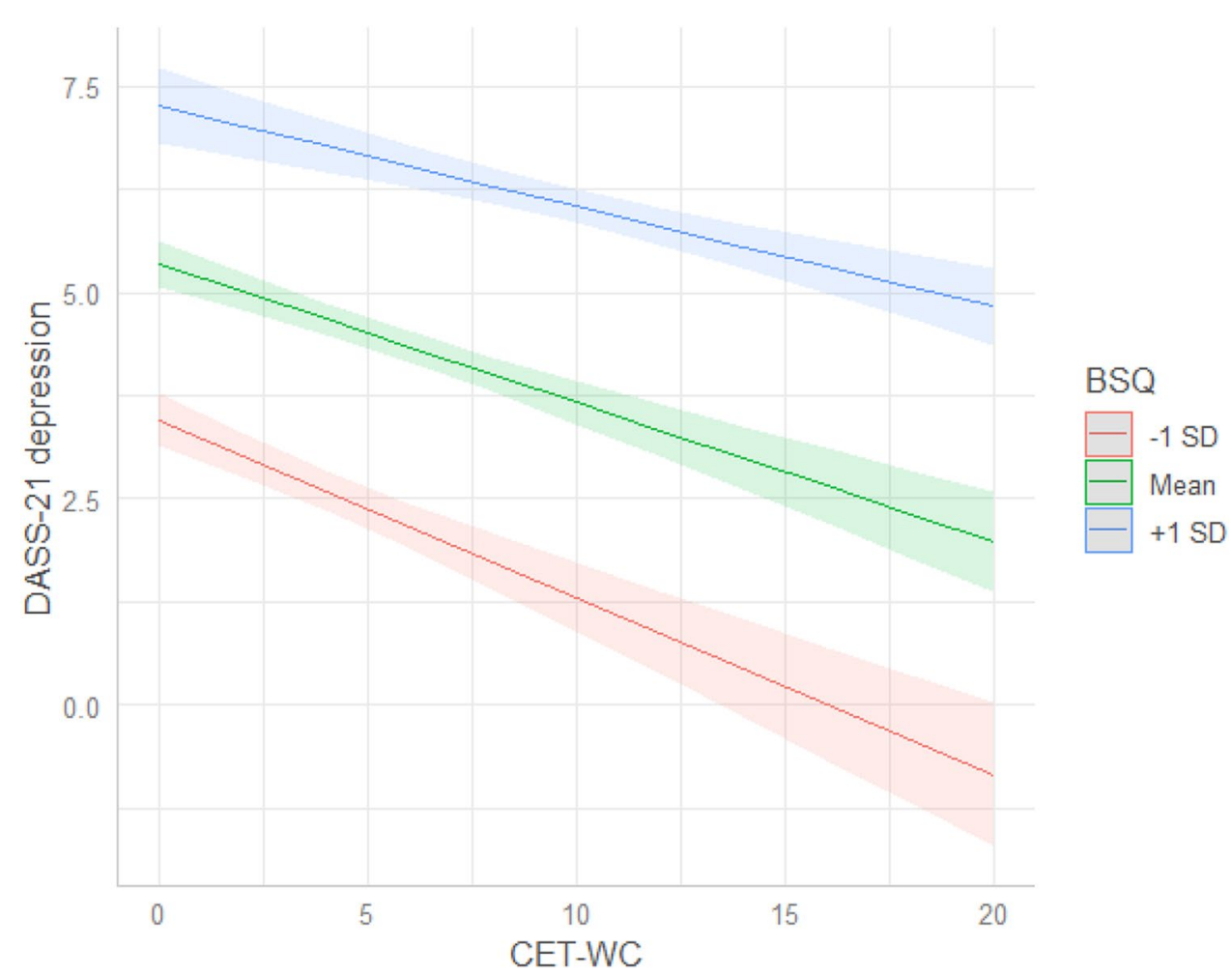

constructs of importance in a non-clinical population regarding self-reported symptoms of depression.

\section{Strengths and limitations}

A strength of the study is that it is based on a large sample from the baseline measure of a cohort study with more than 4000 students included. The use of well-established instruments and an easily accessible digital platform for our web-based surveys are also strengths. A limitation of the study may be that all participating students were enrolled at universities in the Stockholm area which may limit the generalizability of the results. Further, only $23 \%$ of the invited students participated by completing the web-based survey. This is a potential threat to the validity of the study, but since the aim was to study associations, and not to primary report on the occurrence, we believe this potential bias is not extensive.

\section{Clinical relevance}

Body dissatisfaction and compulsive training are core features of eating disorders, and body dissatisfaction has also been considered to increase the risk of developing eating disorders [34, 40, 47, 48]. The co-morbidities with eating disorders and other mental health problems, such as depression, are high. By studying associations between body dissatisfaction, compulsive training, and symptoms of depression, we have identified a positive relation between body dissatisfaction and symptoms of depression along with a dysfunctional strategy for emotion regulation, compulsive training. Our results suggest both constructs to be of interest for reported symptoms of depression in a non-clinical population of Swedish university students, and are, to our knowledge, novel results.

\section{Future research and implications}

To fully understand the relationship between body dissatisfaction and physical activity, further research is needed to understand the potential relationship between not only body dissatisfaction but also positive body image and physical activity [27]. Output from such research may help differentiate between functional and dysfunctional behaviors and attitudes related to body image and exercise, which could be of fundamental importance to the understanding of risks for developing psychopathologies.

The cross-sectional design used in the present study does not lead to understanding the direction of the associations between the study variables. Therefore, longitudinal analyses are needed to understand causal associations between compulsive exercise, body dissatisfaction and depression among students. Thus, data included in the present study are from the baseline measure of the ongoing cohort study SUN, lending the opportunity for further 
analyses to study changes in these variables prospectively over a one-year-period of time.

What is already known on this subject? That body dissatisfaction is a core clinical feature in eating disorders and a well-established risk factor for the development of pathological eating patterns and disorders. What does the study add? The indications of the association between body dissatisfaction, compulsive training, and depression in a non-clinical population of university students. Further that compulsive training was negatively associated with depression suggesting compulsive training to serve as a dysfunctional strategy for emotion regulation.

\section{Conclusion}

In line with previous research, there was a gender difference in body dissatisfaction where females displayed higher levels of dissatisfaction than males. Body dissatisfaction was positively associated with symptoms of depression, suggesting support of previous research indicating body dissatisfaction to increase mental health problems. Compulsive exercise was negatively associated with symptoms of depression suggesting a behavior negatively reinforced, supporting both constructs to be of interest for reported symptoms of depression in a non-clinical population of Swedish university students.

Acknowledgements We would like to thank Clara Onell, Torsten Wrigley, Felix Sjöstrand, Madeleine Naidoo Bjørnstad, ACTIC (health club chain) and Tim Bergling Foundation for in different ways contributing to realization of the project.

Author contributions ES is principal investigator of the SUN-study, data collection for the present paper was performed by KE, ES, FJ, TS, RL and LB along with colleagues mentioned in acknowledgements below. Statical analyses, interpretation and preparation of the manuscript were performed by KE, FJ, ES, RL, LB and TS. All authors provided critical revisions of the intellectual content. The SUN-study is more carefully presented in our study protocol (Edlund, et al., submitted).

Funding Open access funding provided by Sophiahemmet University College. This research project was funded by the Swedish Research Council for Health, Working Life and Welfare (FORTE), Grant Number FORTE2018-00402. The project also received financial support from the Public Health Agency of Sweden.

Availability of data and materials The dataset generated and analyzed during the current study are not publicly available due to secondary confidentiality and privacy of the participants.

\section{Declarations}

Conflict of interest The authors declare that there are no conflicts of interest.
Ethical considerations Prior to the data collection, the Universities' Educational Support Offices and Student Health Services were informed about the study and granted approval to implement the study at each site. Before inclusion in the study, informed consent was given by all participants. The study was approved by the Swedish Ethical Review Authority (Reference Number: 2019-03276; 2020-01449)

Open Access This article is licensed under a Creative Commons Attribution 4.0 International License, which permits use, sharing, adaptation, distribution and reproduction in any medium or format, as long as you give appropriate credit to the original author(s) and the source, provide a link to the Creative Commons licence, and indicate if changes were made. The images or other third party material in this article are included in the article's Creative Commons licence, unless indicated otherwise in a credit line to the material. If material is not included in the article's Creative Commons licence and your intended use is not permitted by statutory regulation or exceeds the permitted use, you will need to obtain permission directly from the copyright holder. To view a copy of this licence, visit http://creativecommons.org/licenses/by/4.0/.

\section{References}

1. Akram U, Ypsilanti A, Gardani M, Irvine K, Allen S, Akram A et al (2020) Prevalence and psychiatric correlates of suicidal ideation in UK university students. J Affect Disord 272:191197. https://doi.org/10.1016/j.jad.2020.03.185 (Epub 2020 May 7)

2. Alfonsson S, Wallin E, Maathz P (2017) Factor structure and validity of the Depression, Anxiety and Stress Scale-21 in Swedish translation. J Psychiatr Ment Health Nurs 24:154-162. https://doi.org/10.1111/jpm.12363

3. Auerbach RP, Alonso J, Axinn WG, Cuijpers P, Ebert DJG et al (2016) Mental disorders among college students in the World Health Organization World Mental Health Surveys. Psychol Med 46:2955-2970

4. Barnes M, Abhyankar P, Dimova E, Best C (2020) Association between body dissatisfaction and self-reported anxiety and depression in otherwise healthy men: a systematic review and meta-analysis. PLoS ONE 15(2):e0229268. https://doi.org/10. 1371/journal.pone.0229268

5. Bassett-Gunter R, McEwan D, Kamarhie A (2017) Physical activity and body image among men and boys: a meta-analysis. Body Image 22:114-128. https://doi.org/10.1016/j.bodyim.2017.06.007

6. Bearman SK, Stice E (2008) Testing a gender additive model: the role of body image in adolescent depression. J Abnorm Child Psychol 36:1251-1263

7. Biddle S, Fox KR, Boutcher SH (2000) Physical activity and psychological well-being. Routledge, London

8. Bornioli A, Lewis-Smith H, Slater A, Bray I (2021) Body dissatisfaction predicts the onset of depression among adolescent females and males: a prospective study. J Epidemiol Community Health 75:343-348. https://doi.org/10.1136/jech-2019-213033

9. Bornioli A, Lewis-Smith H, Smith A et al (2019) Adolescent body dissatisfaction and disordered eating: predictors of later risky health behaviours. Soc Sci Med 238:112458

10. Cooper PJ, Taylor MJ, Cooper Z, Fairburn CG (1986) The development and validation of the Body Shape Questionnaire. Int J Eat Disord 6:485-494

11. Crow S, Eisenberg ME, Story M, Neumark-Sztainer D (2008) Are body dissatisfaction, eating disturbance, and body mass index predictors of suicidal behavior in adolescents? A longitudinal study. J Consult Clin Psychol 76:887-92. https://doi.org/ $10.1037 / \mathrm{a} 0012783$ 
12. Dreier MJ, Dyrbye L, Thomas LN, Shanafelt TD (2006) Systematic review of depression, anxiety and other indicators of psychologic distress among US and Canadian medical students. Acad Med 81:354-373. https://doi.org/10.1097/00001888200604000-00009

13. Dyrbye LN, Thomas MR, Shanafelt TD (2006) Systematic review of depression, anxiety, and other indicators of psychological distress among U.S. and Canadian medical students. Acad Med 81:354-373

14. Edlund, K., Sundberg, T., Côté, P., Rudman, A., Holm, L., Gustavsson, P., Grotle, M., Jensen, I., \& Skillgate, E. The sustainable university life (SUN) study: Protocol for a prospective cohort study of modifiable risk and prognostic factors for mental health problems and musculoskeletal pain among Swedish university students. Submitted.

15. Evans C, Dolan B (1993) Body Shape Questionnaire: derivation of shortened "alternateforms." Int J Eat Disord 13:315-321

16. Ferreiro F, Seoane G, Senra C (2014) Toward understanding the role of body dissatisfaction in the gender differences in depressive symptoms and disordered eating: a longitudinal study during adolescence. J Adolesc 37:73-84

17. Ferreiro F, Seoane G, Senra C (2012) Sex-related risk and protective factors for depressive symptoms and disordered eating in adolescence: a 4-year longitudinal study. J Youth Adolesc 41:607-622

18. Formby P, Watson HJ, Hilyard A, Martin K, Egan SJ (2014) Psychometric properties of the Compulsive Exercise Test in an adolescent eating disorder population. Eat Behav 15:555-557

19. Frögeli E, Rudman A, Gustavsson P (2019) The relationship between task mastery, role clarity, social acceptance, and stress: an intensive longitudinal study with a sample of newly registered nurses. Int J Nurs Stud 91:60-69

20. Ganson KT, Mitchison D, Rodgers RF, Cunningham ML, Murray SB, Nagata JM (2021) Compulsive exercise among college students: 5-year time trends in prevalence and demographic, substance use, and mental health correlates. Eat Weight Disord. https://doi.org/10.1007/s40519-021-01210-8

21. Girard M, Rodgers RF, Chabrol H (2018) Prospective predictors of body dissatisfaction, drive for thinness, and muscularity concerns among young women in France: a sociocultural model. Body Image 26:103-110. https://doi.org/10.1016/j.bodyim.2018. 07.001

22. Goldschmidt AB, Wall M, Choo TH et al (2016) Shared risk factors for mood-, eating-, and weight-related health outcomes. Health Psychol 35:245

23. Heider N, Spruyt A, De Houwer J (2018) Body dissatisfaction revisited: on the importance of implicit beliefs about actual and ideal body image. Psychol Belg 57:158-173. https://doi.org/10. 5334/pb.362

24. Holland G, Tiggemann M (2017) "Strong beats skinny every time": disordered eating and compulsive exercise in women who post fitspiration on Instagram. Int J Eat Disord 50:76-79. https:// doi.org/10.1002/eat.22559

25. Ibrahim AK, Kelly SJ, Adams CE, Glazebrook C (2013) A systematic review of studies of depression prevalence in university students. J Psychiatr Res 47:391-400

26. Ivarsson T, Svalander P, Litlere O, Nevonen L (2006) Weight concerns, body image, depression and anxiety in Swedish adolescents. Eat Behav 7:161-175

27. Jankauskiene R, Baceviciene M, Trinkuniene T (2020) Examining body appreciation and disordered eating in adolescents of different sports practice: cross-sectional study. Int J Environ Res Public Health 17:4044. https://doi.org/10.3390/ijerph17114044

28. Kelley Galliger CC, Neufeld JM, Musher-Eizenman DR (2010) Drive for thinness and drive for muscularity: opposite ends of the continuum or separate constructs? Body Image 7:74-77
29. Kling J, Kwakkenbos L, Diedrichs PC, Rumsey N, Frisén A, Brandão MP, Silva AG, Dooley B, Rodgers RF, Fitzgerald A (2019) Systematic review of body image measures. Body Image 30:170-211

30. Lageborn CT et al (2017) Ongoing university studies and the risk of suicide: a register-based nationwide cohort study of 5 million young and middle-aged individuals in Sweden, 1993-2011. BMJ Open. https://doi.org/10.1136/bmjopen-2016-014264

31. Lichtenstein MB, Hinze CJ, Emborg B, Thomsen F, Hemmingsen SD (2017) Compulsive exercise: links, risks and challenges faced. Psychol Res Behav Manag 10:85-95

32. Lipson SK, Sonneville KR (2020) Understanding suicide risk and eating disorders in college student populations: results from a National Study. Int J Eat Disord 53:229-238

33. Lovibond PF, Lovibond SH (1995) The structure of negative emotional states: comparison of the Depression Anxiety Stress Scales (DASS) with the Beck Depression and Anxiety Inventories. Behav Res Ther 33:335-343

34. McLean S, Paxton SJ (2019) Body image in the context of eating disorders. Psychiatr Clin North Am 42:145-156. https://doi.org/ 10.1016/j.psc.2018.10.006

35. Meyer C, Taranis L, Goodwin H, Haycraft E (2011) Compulsive exercise and eating disorders. Eur Eat Disord Rev 19:174-189

36. Meyer C, Taranis L (2011) Exercise in the eating disorders: terms and definitions. Eur Eat Disord Rev 19:169-173. https://doi.org/ 10.1002/erv.1121

37. Mond J, Gorrell S (2021) "Excessive exercise" in eating disorders research: problems of definition and perspective. Eat Weight Disord Stud Anorexia Bulim Obes. https://doi.org/10.1007/ s40519-020-01075-3

38. Mond J, Van den Berg P, Boutelle K et al (2011) Obesity, body dissatisfaction, and emotional well-being in early and late adolescence: findings from the project EAT study. J Adolescent Health 48:373-378

39. Murray SB, Nagata JM, Griffiths S, Calzo JP, Brown TA, Mitchison DD et al (2017) The enigma of male eating disorders: a critical review and synthesis. Clin Psychol Rev 57:1-11. https://doi. org/10.1016/j.cpr.2017.08.001

40. Neumark-Sztainer D, Paxton SJ, Hannan PJ et al (2006) Does body satisfaction matter? Five- year longitudinal associations between body satisfaction and health behaviors in adolescent females and males. J Adolescent Health 39:244-251

41. O'Hara SE, Cox AE, Amorose AJ (2014) Emphasizing appearance versus health outcomes in exercise: the influence of the instructor and participants' reason for exercise. Body Image 11:109-118

42. Paxton SJ, Neumark-Sztainer D, Hannan PJ et al (2006) Body dissatisfaction prospectively predicts depressive mood and low self-esteem in adolescent girls and boys. J Clin Child Adolescent Psychol 35:539-549

43. Penas-Lledo E, Vaz Leal F, Waller G (2002) Excessive exercise in anorexia nervosa and bulimia nervosa: relation to eating characteristics and general psychopathology. Int J Eat Disord 31:370375. https://doi.org/10.1002/eat.10042

44. Ricciardelli LA, McCabe MP (2001) Children's body image concerns and eating disturbance. Clin Psychol Rev 21:325-344. https://doi.org/10.1016/S0272-7358(99)00051-3

45. Rodgers RF, Franko DL, Lovering ME, Luk S, Pernal W, Matsumoto A (2018) Development and validation of the female muscularity scale. Sex Roles 78:18-26. https://doi.org/10.1007/ s11199-017-0775-6

46. Schaumberg K, Anderson LM, Reilly E, Anderson DA (2014) Patterns of compensatory behaviors and disordered eating in college students. J Am Coll Heal 62:526-533. https://doi.org/10.1080/ 07448481.2014 .930468

47. Stice E, Gau JM, Rohde P, Shaw H (2017) Risk factors that predict future onset of each DSM-5 eating disorder: Predictive specificity 
in high-risk adolescent females. J Abnorm Psychol 126:38-51. https://doi.org/10.1037/abn0000219

48. Stice E, Shaw HE (2002) Role of body dissatisfaction in the onset and maintenance of eating pathology: a synthesis of research findings. J Psychosom Res 53:985-993

49. Stice E, Bearman SK (2001) Body-image and eating disturbances prospectively predict increases in depressive symptoms in adolescent girls: a growth curve analysis. Dev Psychol 37:597

50. Stice E, Hayward C, Cameron RP et al (2000) Body-image and eating disturbances predict onset of depression among female adolescents: a longitudinal study. J Abnorm Psychol 109:438

51. Stoeber J, Otto K (2006) Positive conceptions of perfectionism: approaches, evidence, challenges. Pers Soc Psychol Rev 10:295-319

52. Strömbäck M, Wiklund M, Salander Renberg E, Malmgren-Olsson EB (2015) Complex symptomatology among young women who present with stress-related problems. Scand J Caring Sci 29:234-247

53. SUNET. Skapa enkäter med SUNET Survey [Internet]. 2019 []. Available from: https://www.sunet.se/tjanster/survey/. Cited 6 Dec 2019

54. Swami V, Frederick DA, Aavik T et al (2010) The attractive female body weight and female body dissatisfaction in 26 countries across 10 world regions: results of the international body project I. Pers Soc Psychol Bull 36:309-325

55. Taranis L, Touyz S, Meyer C (2011) Disordered eating and exercise: development and preliminary validation of the compulsive exercise test (CET). Eur Eat Disord Rev 19:256-268

56. Taylor, J. T. (1987). The nature and significance of body image disturbance. Wolfson College. University of Cambridge for the
Degree of Doctor of Philosophy. https://www.psyctc.org/psyctc/ wp-content/uploads/2021/01/BSQ_PhD_thesis_Taylor_1987.pdf

57. The Children's Society (2020). The good childhood report 2016. 2012. Available: https://www.childrenssociety.org.uk/sites/defau 1t/files/pcr090_mainreport_web.pdf. Accessed 19 Feb 2020

58. Thompson JK, Heinberg LJ, Altabe M et al (1999) Exacting beauty: theory, assessment, and treatment of body image disturbance. American Psychological Association, Washington

59. Vrabel K, Bratland-Sanda S (2019) Exercise obsession and compulsion in adults with longstanding eating disorders: validation of the Norwegian version of the Compulsive exercise test. Front Psychol 10:2370

60. Weinstein A, Maayan G, Weinstein Y (2015) A study on the relationship between compulsive exercise, depression and anxiety. $\mathrm{J}$ Behav Addict 4:315-318

61. Welch E, Lagerström M, Ghaderi A (2012) Body Shape Questionnaire: psychometric properties of the short version (BSQ-8C) and norms from the general Swedish population. Body Image $10: 547-550$

62. Wertheim EH, Paxton SJ (2012) Body image development-adolescent girls. In: Cash TF (ed) Encyclopedia of body image and human appearance. Elsevier, Amsterdam, pp 187-193. https://doi. org/10.1016/B978-0-12-384925-0.00029-8

Publisher's Note Springer Nature remains neutral with regard to jurisdictional claims in published maps and institutional affiliations. 